\title{
Subtotal Esophagectomy-Akiyama Procedure: In a Case with Esophageal Squamous Cell Carcinoma
}

\author{
Henri Kolani Islam Mamica Eriol Braholli Etmont Çeliku \\ University Hospital Center "Mother Theresa", Tirana, Albania, Albania
}

\begin{abstract}
Adenocarcinoma, typically in the distal third of the esophagus, and squamous cell carcinoma, typically in the proximal two thirds of the esophagus, each make up $49 \%$ of cases of esophageal cancer. The remaining cancers in this area include sarcoma $(1 \%)$, lymphoma $(0.5 \%)$, cylindroma $(0.25 \%)$, and primary melanoma $(0.25 \%)$.

Case report

We present patient S.G. 62 years old, male, with the following symptoms started 3 months from hospital admission: difficult swalloing mostly for the hard foods and eventually for the liquids too, chest burning, cough and vomiting, throat pain, weight loss. In the laboratory findings only a mild anemia was found, Hemoglobine $10.8 \mathrm{~g} / \mathrm{dl}$, and slightly high values of CRP. Patient was hospitalized in the First Clinic of General Surgery, University Hospital Center "Mother Teresa" Tirana, Albania and underwent an upper gastrointestinal tract endoscopy and $28 \mathrm{~cm}$ form the incisive teeth and esophageal narrowing was detected which could not allow the scope to go lower for further examination. Biopsy was taken through endoscopy. Patient was planned for a CT scan and tumor markers, CEA and CA 19-9, both later ones came in normal values. CT scan showed an irregular, asymmetric narrowing of the thoracic esophagus, thickening of the esophagus walls with $4 \mathrm{~cm}$ of extension without invasion of local periesophageal fat and regional lymphadenopathy. The biopsy resulted; esophageal squamous cell carcinoma G2. In these circumstances patient underwent a feeding jejunostomy and was sent to follow the protocol of neoadjuvant chemo and radiotherapy. Three months later the patient is returned in our clinic, where he underwent the surgical intervention. Postoperatively the patient was treated in the Intensive Care Unit. The next day after the operation, cervical drains were removed and in the fourth postoperative day the thoracic and abdominal drains were also removed. Patient comes in the surgery ward in the fifth postoperative day where is treated afterwards with an excellent postoperative course. In the tenth postoperative day the anastomosis integrity is verified by $\mathrm{x}$-ray swallowing contrast gastrografin, and the next day he was discharged from the hospital.
\end{abstract}

Keywords: Esophageal, squamous cell carcinoma, subtotal esophagectomy

DOI: $10.7176 /$ ALST/79-05

Publication date:March $31^{\text {st }} 2020$

\section{Introduction}

The incidence of adenocarcinoma is clearly increasing; it will soon become the most prevalent type of cancer of the esophagus. No malignant tumor in the past 25 years has increased in incidence as much as adenocarcinoma of the esophagus. ${ }^{1}$ The primary risk of adenocarcinoma is related to the duration and severity of gastroesophageal reflux and the progression of mucosal changes from Barrett's esophagus to dysplasia to adenocarcinoma.The incidence of squamous cell carcinoma, which used to be the major cause of esophageal cancer, has significantly decreased. ${ }^{1}$ The decrease may be related to reductions in risk factors, which include smoking, excessive alcohol use, caustic lye injury or thermal injury, diet, obesity, achalasia, and tylosis. ${ }^{1}$ Typical symptoms of esophageal cancer include difficulty in swallowing, with a feeling of fullness, pressure, burning, or coughing, a feeling of both liquids and solids becoming stuck behind the sternum, indigestion, emesis and weight loss.

\section{Diagnostic methods}

Endoscopy and biopsy are essential for determining whether the tumor is squamous cell carcinoma, Barrett's esophagus, or adenocarcinoma. However, multiple staging modalities are available, from physical examination to computed tomography (CT), endoscopic ultrasound, magnetic resonance imaging (MRI), positron emission tomography (PET), thoracoscopy/laparoscopy, bone scan, laryngoscopy and bronchoscopy. ${ }^{2-10} \mathrm{CT}$ scan is used most frequently in diagnosis. While it clearly shows the presence of tumor, it is only $49 \%$ to $60 \%$ accurate in staging the depth of the tumor, according to published reports. ${ }^{7.8 .9 .10}$ Endoscopic ultrasound is significantly better in determining $\mathrm{T}$ stage, with an accuracy ranging from $76 \%$ to $92 \%{ }^{3,4,5,6,7}$ While few studies have examined MRI's success in determining $\mathrm{T}$ stage, it appears to be very promising, with an accuracy rate of $96 \%$ to $100 \%{ }^{10}$

\section{Material and methods}

During last year, from October 2018 to October 2019, 15 operations for esophageal and gastroesophageal junction cancer are performed in The First Surgical Clinic, UHC "Mother Teresa". In 7 patients, subtotal esophagectomy Ivor-Lewis with a mediastinal esophago-gastric anastomosis was performed. In 4 patients, distal esophagectomy and proximal gastrectomy with an abdominal esophago-gastric anastomosis was performed. In 3 patients, distal 
esophagectomy and total gastrectomy with Roux-en-Y esophago-jejunostomy reconstruction was performed. In 1 patient, subtotal esophagectomy with a cervical esophago-gastric anastomosis (Akiyama Procedure) was performed.

\section{Case report}

We present patient S.G. 62 years old, male, with following symptoms started 3 months previous hospital admission: swallowing difficulties mostly for hard foods and actually for the liquids as well, chest burning, cough, vomiting, throat pain and weight loss. In the laboratory findings only a mild anemia was found, Hemoglobine $10.8 \mathrm{~g} / \mathrm{dl}$, and slightly high values of CRP.

The patient was hospitalized in the First Clinic of General Surgery, in University Hospital Center "Mother Teresa", Tirana, Albania and underwent an upper gastrointestinal tract endoscopy (figure 1), where $28 \mathrm{~cm}$ form the incisive teeth an esophageal narrowing was detected which could not allow the scope to go lower for further examination.

Biopsy was taken through endoscopy.

Menagement options

For resection, surgeons use several techniques:

the IvorLewis, a thoracoabdominal approach;

the transhiatal, involving the abdomen and neck while avoiding the thoracic incision;

the transabdominal, used particularly for cancers of the lower gastroesophageal junction;

total thoracic esophagectomy McKeown or Akiyama procedure;

the thoracoscopic/laparoscopic, a minimally invasive approach.

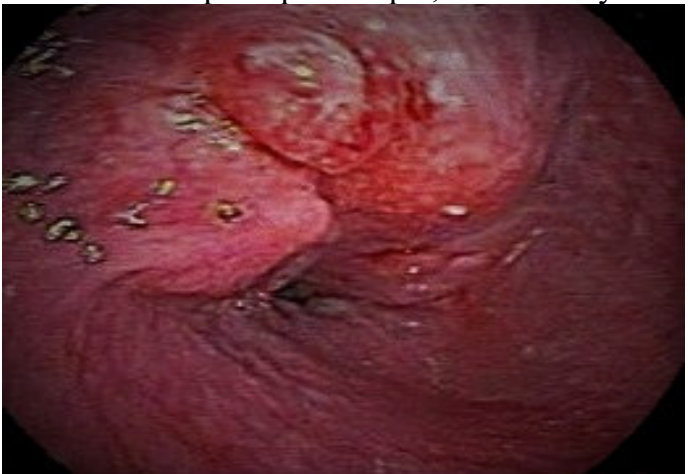

Figure 1. Endoscopy view

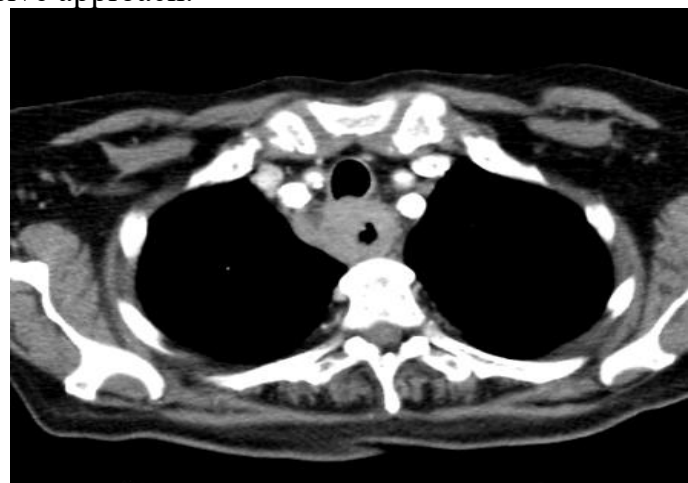

Figure 2. CT scan of $\mathrm{t}$

After that the patient was planned for a CT scan and tumor markers, CEA and CA 19-9. Both of them came in normal values. CT scan showed an irregular, asymmetric narrowing of the thoracic esophagus, thickening of the esophagus walls with $4 \mathrm{~cm}$ of extension without invasion of local periesophageal fat and regional lymphadenopathy. The biopsy resulted in esophageal squamous cell carcinoma G2. In these circumstances patient underwent a feeding jejunostomy and was sent to follow the protocol of neoadjuvant chemo and radiotherapy. Three months later the patient is returned in the First Surgical Clinic where he underwent the surgical intervention. Surgical procedure
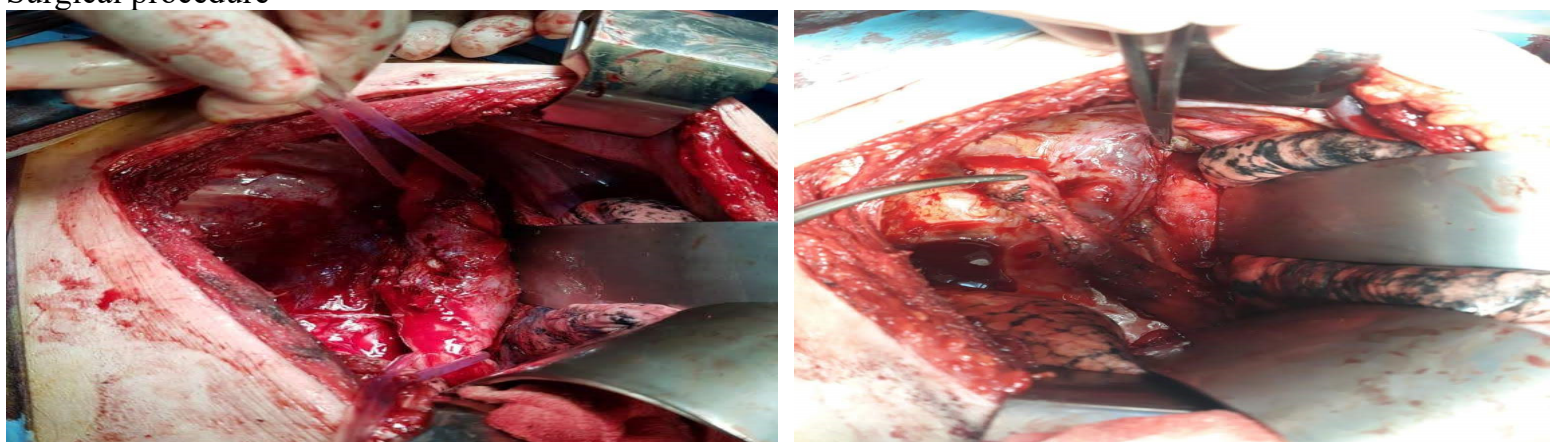

Figure 2, A, B. Subtotal esophageal resection performed, closely to the thoracic inlet, $5 \mathrm{~cm}$ above the tumor. 

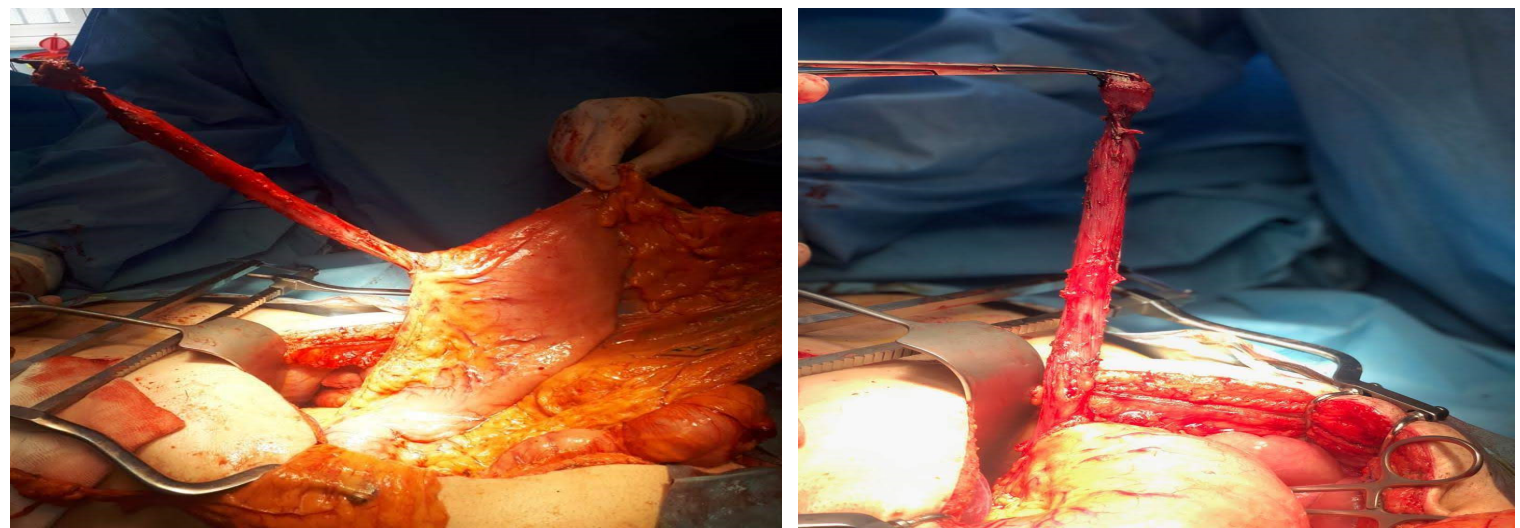

Figure 3. A, B. Surgical procedure continued by a midline abdominal incision and the prepared esophagus was pulled out in the abdomen.
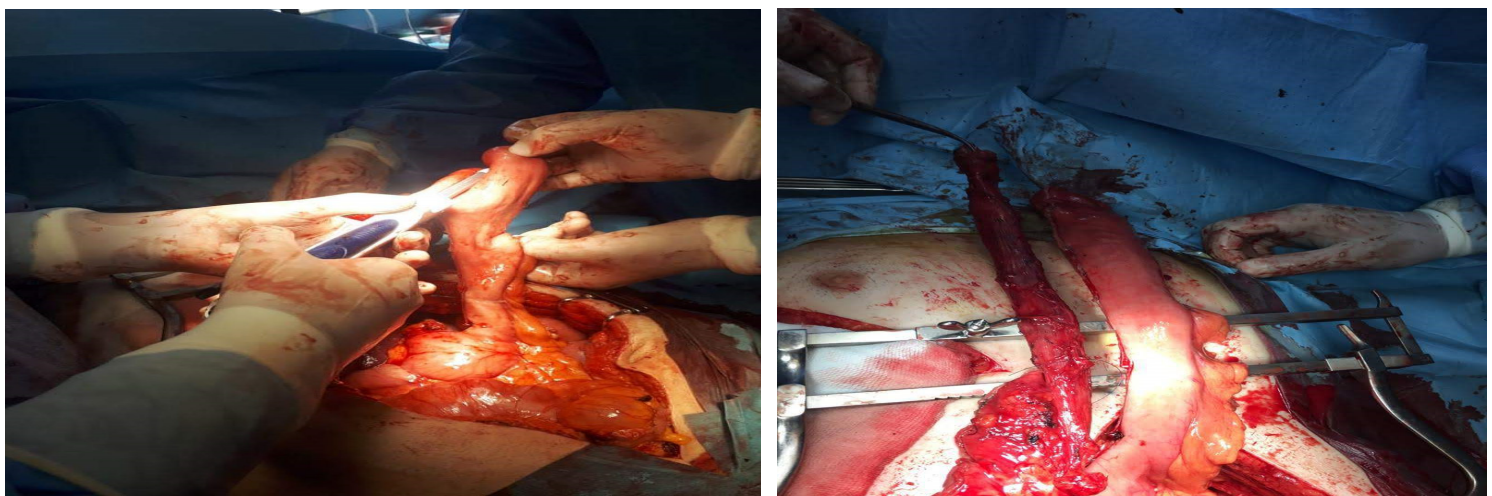

Figure 4. A, B. After that the resection of the lesser curvature was made and the stomach was prepared as a conduit for the esophageal reconstruction followed by Heineke-Miculitz pyloroplasty.
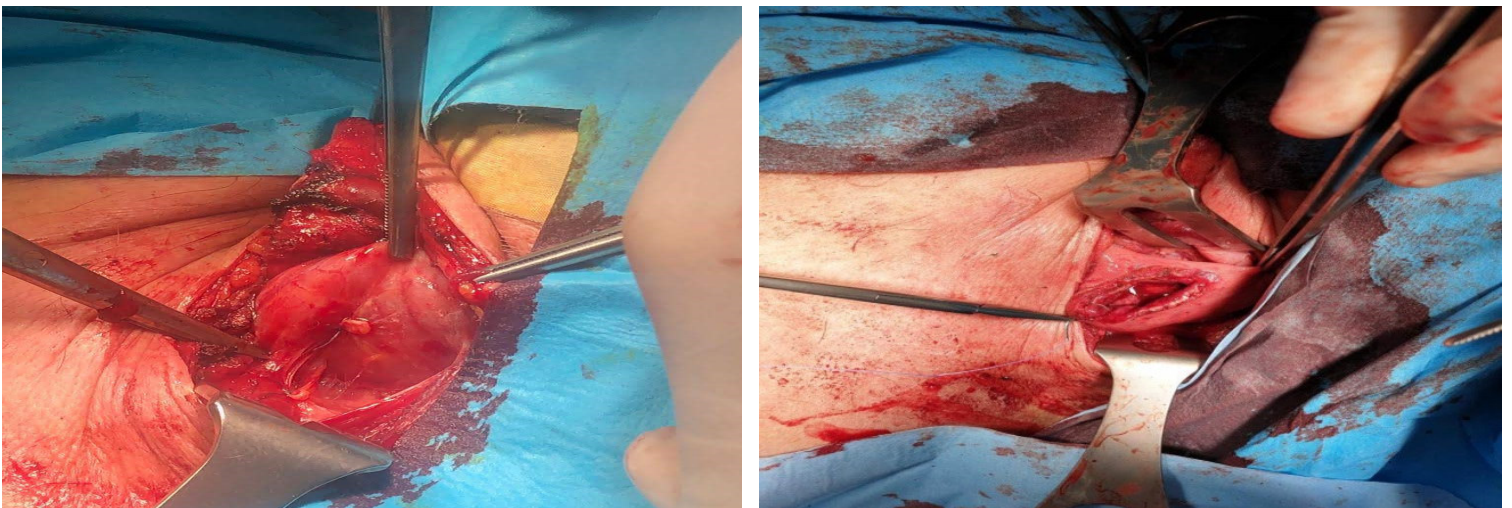

Figure 5. A, B. A third left parasternal incision was done in the neck where cervical esophagus was exposed and the distal end was pulled out. A retrosternal tunnel was created manually and the stomach was delivered in the neck through this tunnel. 

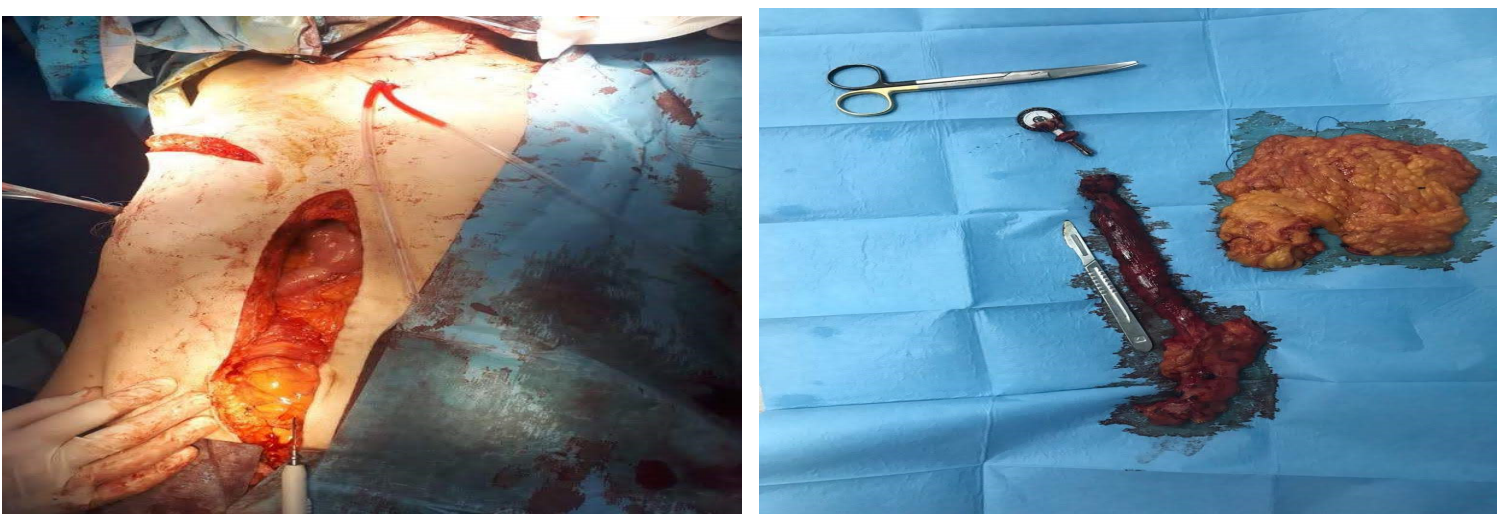

Figure 6. An end to end anastomosis was performed in the neck using a circular stapler $25 \mathrm{~mm}$. So a subtotal esophageal resection with a cervical esophagogastric anastomosis retrosternal positioned (Akiyama Procedure) was performed. Each cavity was closed and drained.
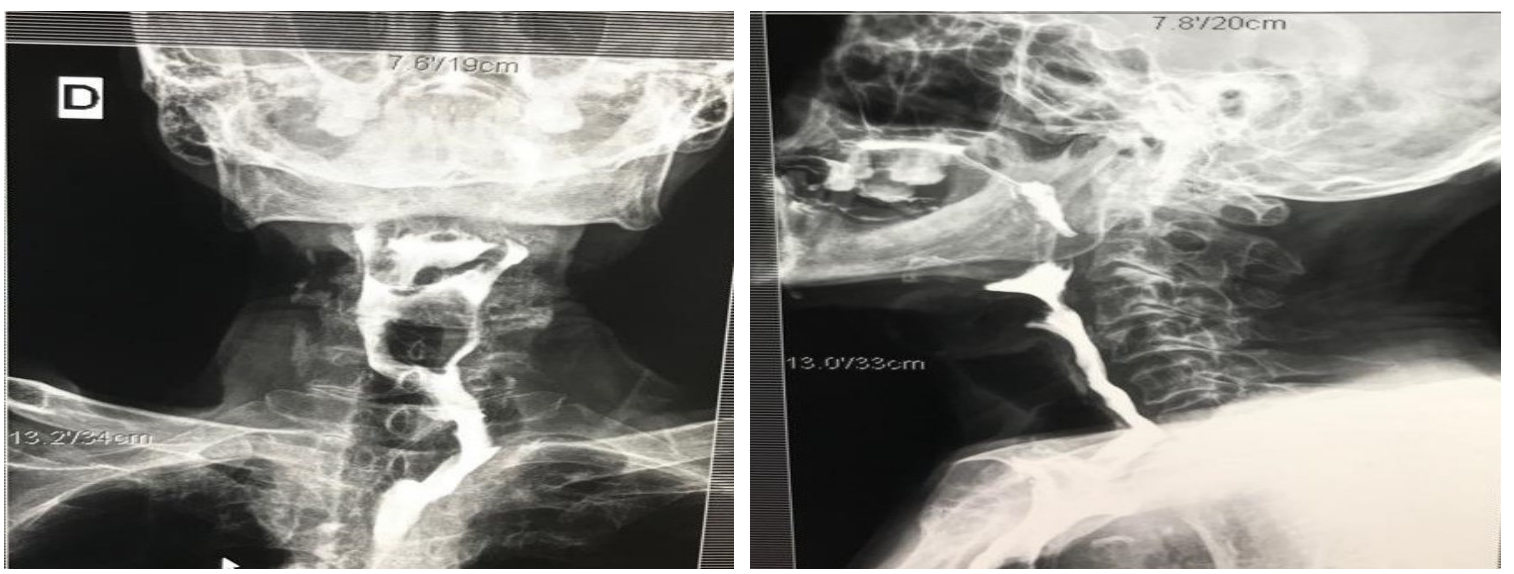

Figure 7. In the tenth postoperative day the anastomosis integrity is verified by $\mathrm{x}$-ray swallowing contrast gastrografin, and the next day he was discharged from the hospital.

\section{Discussion}

Postoperatively the patient was treated in the Intensive Care Unit. The next day after the operation, cervical drains were removed and in the fourth postoperative day the thoracic and abdominal drains were also removed. Patient comes in the surgery ward in the fifth postoperative day where is treated afterwards with an excellent postoperative course. In the tenth postoperative day the anastomosis integrity is verified by $\mathrm{x}$-ray swallowing contrast gastrografin, and the next day he was discharged from the hospital. The biopsy of the specimen resulted squamous cell carcinoma with moderate differentiation G2. All the 5 lymph nodes were reactive, no invasion in the periesophageal fat tissue.

The survival rate of patients undergoing surgery for thoracic esophageal cancer has improved in recent years, partly as a result of advances in surgical techniques and perioperative management. ${ }^{11,12}$ However, this extensive surgery is still associated with high complication rates and a long-standing negative impact on the patient's quality of life (QOL). Early postoperative complications such as anastomotic leakage cause significant morbidity. Thus, reducing the risk of such complications could reduce the negative impact on QOL after esophageal cancer surgery.

Gastric transposition with esophagogastric anastomosis is a common method of reconstruction after esophagectomy. ${ }^{12,13,14,15,16,17}$ Esophagogastric anastomoses can be fashioned in the neck or in the chest, by handsewing or by using a mechanical stapling device. Several investigators have compared these two techniques applied at both sites but controversy still exists on which is the best approach.

In our study subtotal esophagectomy through a right thoracotomy was performed. Stomach was used for esophageal reconstruction. Anastomosis was made in the neck using a circular stapling device. Stomach was delivered in the neck through a retrosternal tunnel made manually. The primary aim of surgical treatment for carcinoma of the esophagus is cure of the disease or prolongation of survival time and the ability to ingest food normally. A balanced combination of resection and reconstruction with minimal surgical risks is important. It has been demonstrated that complete lymph node dissection is necessary in the mediastinum as well as in the abdomen, regardless of the anatomic location of the tumor. ${ }^{18,19,20}$ However, resection is not indicated for patients with extensive tumor involvement in the cervical, para-aortic, retroperitoneal, and or other distant lymph nodes. In our 
case three field lymphnode dissection was made, in the neck, thorax and abdomen. For better exposure of the whole posterior mediastinum, a right thoracotomy should be chosen. In order to have a safe surgical margin, care must be taken, particularly for lesions of the upper esophagus. Esophagotomy in the neck is useful in order to avoid a cancer-positive surgical margin. Anastomosis is routinely performed in the neck. ${ }^{21,22,23}$

\section{Conclusions}

Cervical anastomosis has the advantages of postoperative safety and excellent exposure, which makes the anastomosis accurate. The retrosternal space is routinely used as the route of esophageal replacement in order to avoid the possibility of local tumor recurrence in the posterior mediastinum. However, for early-stage carcinoma, a posterior mediastinal route can be chosen. The presternal route is rarely used, because of length and cosmetic reasons. An intrathoracic anastomosis is not recommended, because of the possibility of incomplete mediastinal node dissection and prolongation of the time of thoracotomy. Preoperative and postoperative irradiation or other adjuvant therapies by chemotherapy or immunotherapy are also important means of treatment. They have been used for selected patients. However, more studies with larger population have to be performed of the effects on patients with carcinoma of the esophagus, for e better understanding.

\section{Bibliography}

1. American Cancer Society . Cancer Facts and Figures 2003. Atlanta, Ga: American Cancer Society; 2003.

2. Massari M, Cioffl U, de Simone M, Lattuada E, Montorsi M, Segalin A, Bonavina L. Endoscopic ultrasonography for pre-operative staging of esophageal carcinoma. Surg Laparosc Endosc. 1997;7:162-165.

3. Hordijk ML, Zander H, van Blankenstein M, Tilanus HW. Influence of tumor stenosis on the accuracy of endosonography in preoperative T staging of esophageal cancer. Endoscopy. 1993;25:171-175.

4. Nattermann C, Dancygier H. Endoscopic ultrasound in preoperative TN staging of esophageal cancer. A comparative study between endosonography and computerized tomography. Ultraschall Med. 1993;14:100105 .

5. Kalantzis N, Kallimanis G, Laoudi F, et al. Endoscopic ultrasonography and computed tomography in preoperative (TNM) classification of oesophageal carcinoma [abstract] Endoscopy. 1992;24:653A.

6. Ziegler K, Sanft C, Zeitz M, Friedrich M, Stein H, Haring R, Riecken EO. Evaluation of endosonography in TN staging of oesophageal cancer. Gut. 1991;32:16-20.

7. Tio TL, Cohen P, Coene PP, Udding J, den Hartog Jager FC, Tytgat GN. Endosonography and computed tomography of esophageal carcinoma. Preoperative classification compared to the new (1987) TNM system. Gastroenterotogy. 1989;96:1478-1486.

8. Fekete F, Sauvanet A, Zins M, Berthoux L, Amouyal G. Imaging of cancer of the esophagus: ultrasoundendoscopy or computed tomography? Ann Chir. 1995;49:573-578.

9. Botet JF, Lightdale CJ, Zauber AG, Gerdes H, Urmacher C, Brennan MF. Preoperative staging of esophageal cancer: comparison of endoscopic US and dynamic CT. Radiology. 1991;181:419-425.

10. Yamada I, Murata Y, Izumi Y, Kawano T, Endo M, Kuroiwa T, Shibuya H. Staging of esophageal carcinoma in vitro with 4-7' TMR imaging. Radiology. 1997;204:521-526.

11. Ando, N, Ozawa, S, Kitagawa, Y, Shinozawa, Y, Kitajima, M 2000Improvement in the results of surgical treatment of advanced squamous esophageal carcinoma during 15 consecutive yearsAnn Surg 232:225-32

12. Abo, S, Kitamura, M, Hashimoto, M, Izumi, K, Minamiya, Y, Shikama, T, et al. 1996Analysis of results of surgery performed over a 20-year period on 500 patients with cancer of the thoracic esophagus Surg Today 26:77-82

13. Blazeby, JM, Farndon, JR, Donovan, J, Alderson, D 2000 A prospective longitudinal study examining the quality of life of patients with esophageal carcinoma Cancer 88:1781-7

14. Viklund, P, Lindblad, M, Lagergren, J 2005Influence of surgery-related factors on quality of life after esophageal or cardia cancer resection World J Surg 29:841-8

15. Walther, B, Johansson, J, Johnsson, F, Von Holstein, CS, Zilling, T 2003 Cervical or thoracic anastomosis after esophageal resection and gastric tube reconstruction: a prospective randomized trial comparing sutured neck anastomosis with stapled intrathoracic anastomosis Ann Surg 238:803-12

16. Laterza, E, de' Manzoni, G, Veraldi, GF, Guglielmi, A, Tedesco, P, Cordiano, C 1999 Manual compared with mechanical cervical oesophagogastric anastomosis: a randomised trialEur J Surg 165:1051-4

17. Law, S, Fok, M, Chu, KM, Wong, J 1997 Comparison of hand-sewn and stapled esophagogastric anastomosis after esophageal resection for cancer: a prospective randomized controlled trial Ann Surg 226:169-73

18. Craig, SR, Walker, WS, Cameron, EW, Wightman, AJ 1996A prospective randomized study comparing stapled with handsewn oesophagogastric anastomoses J R Coll Surg Edinb 41:17-19

19. Valverde, A, Hay, JM, Fingerhut, A, Elhadad, A 1996 Manual versus mechanical esophagogastric anastomosis after resection for carcinoma: a controlled trial. French Associations for Surgical Research Surgery 120:476-83 
20. Hsu, HH, Chen, JS, Huang, PM, Lee, JM, Lee, YC 2004 Comparison of manual and mechanical cervical esophagogastric anastomosis after esophageal resection for squamous cell carcinoma: a prospective randomized controlled trial Eur J Cardiothorac Surg 25:1097-101

21. Blewett, CJ, Miller, JD, Young, JE, Bennett, WF, Urschel, JD 2001Anastomotic leaks after esophagectomy for esophageal cancer: a comparison of thoracic and cervical anastomosesAnn Thorac Cardiovasc Surg 7:758

22. Schmidt, CE, Bestmann, B, Kuchler, T, Schmid, A, Kremer, B 2004Quality of life associated with surgery for esophageal cancer: differences between collar and intrathoracic anastomoses World J Surg 28:355-60

23. Akiyama H. Surgery for carcinoma of the esophagus. Curr Probl Surg 1980; XVII: 101 